\title{
Including management and security of supply constraints for designing stand-alone electrification systems in developing countries
}

\author{
Domenech B $^{\mathrm{ac} *}$, Ferrer-Martí $\mathrm{L}^{\mathrm{bc}}$, Pastor $\mathrm{R}^{\mathrm{ac}}$ \\ ${ }^{a}$ Department of Management (DOE) \\ ${ }^{\mathrm{b}}$ Department of Mechanical Engineering (DEM) \\ ${ }^{\mathrm{c}}$ Institute of Industrial and Control Engineering (IOC) \\ Universitat Politècnica de Catalunya; Av. Diagonal 647, pavilion F - floor 0, 08028 Barcelona (Spain). \\ Contact (*): bruno.domenech@upc.edu; (+34) 934016579.
}

\begin{abstract}
Hybrid wind-photovoltaic stand-alone systems have proven to be suitable to electrify isolated communities autonomously. Moreover, the use of a combination of microgrids and individual systems has been demonstrated to be very adequate. There are a few tools to assist their design but they only consider economical and technical characteristics. However, the management of the system and the security of supply, both at a community level, are key aspects to design appropriate electrification systems for end-users, thus ensuring projects' long-term sustainability, especially in rural areas of developing countries. In this context, this paper develops a mathematical model to optimise the design of wind-photovoltaic projects combining microgrids and individual systems, and including the aforesaid key issues as constraints. Thus, the aim is to minimise the cost while meeting the technical but also the management and the security of supply constraints. Finally a validation is carried out in the real community of Alto Peru (Peru), proving that the two studied aspects allow obtaining electrification solutions with some benefits that strongly compensate the obtained slight cost increases.
\end{abstract}

Keywords: wind-photovoltaic; stand-alone electrification; management; supply security.

\section{Introduction}

At present, around 1.3 billion people lack access to electricity [1], especially in rural areas of developing countries [2]. The conventional strategy for providing electricity consists on extending the national grid. However, this strategy presents limitations when reaching rural or mountainous areas with scattered population. Alternatively stand-alone electrification systems based on the use of renewable energies are increasingly being used [3], since they help improving projects' sustainability using local resources and avoiding external dependencies [4]. In particular, the use of a combination of wind and photovoltaic (PV) technologies is significantly increasing, since both complement to each other in relation to continuity of supply and allow saving costs [5].

Given the characteristic dispersion between households in rural communities of developing countries, the common electrification solution consists on installing individual systems: independent generation, storage and distribution equipment are installed to supply each point [6]. Alternatively, microgrids are increasingly been used. This is an electric configuration where the generation and storage equipment are concentrated at a single point and the electricity is then distributed to a set of points. The advantages of microgrids are significant [7,8]: the consumption of a point is not conditioned by the energy resources at its location, the equality in consumption between users is favoured, costs can be saved due to economies of scale and the flexibility in consumption is promoted (allowing punctual increases thanks to the greater amount of 
available energy). However, microgrids entail a higher design difficulty: their structure and connections have to be planned, and a good compromise between their extension and the possible cost increase has to be studied [9]. For this reason, a decision-aid model is needed to assist the design of such systems.

Literature related to the design of stand-alone electrification systems, mainly focuses on combining technologies to meet a specific demand, without studying the detail of the electric distribution configuration [10]. Very few papers overcome these limitations, especially when studying rural areas of developing countries. Ter-Gazarian and Kagan [11] design a model that sizes and locates the generation and storage equipment, but only considering one microgrid without individual systems. VIPOR [12] draws on the results from HOMER [13] to design the distribution system combining microgrids and individual systems, but does not consider different energy resources for the individual systems and does not include voltage drops. Finally, Ferrer-Martí et al. [14] develop a mathematical model for designing stand-alone electrification systems with wind-PV technologies, considering the detail of the energy resources, the energy and power demands at each consumption point and obtaining, as a solution, the emplacement of all the equipment to implement, and the adequate combination of microgrids (with their structure) and individual systems.

The previous publications design the electrification systems only considering economical and technical characteristics. Other works focus on additional aspects, which are key factors to ensure projects' long-term sustainability, which is always an issue in rural areas of developing countries [15]. Although such aspects could be included as constraints, they have usually been considered in literature as design criteria. In this way, Wang et al. [16] review common criteria used in literature when designing electrification systems: social acceptability, job creation and social benefits; which can have different implications depending on the context or the region. In largescale works, they tend to have economic implications [17], as the labour impact or the market maturity; environmental implications [18], as the visual impact or the acoustic noise; or institutional implications [19], as the cohesion to local activities. However, in rural areas of developing countries, a higher detail of criteria at a community level is required in order to adapt the project to the population characteristics [20]. For example, SURE distinguishes, among others, between social criteria, related to the community organisation, and human criteria, related to the education and health [21]. Biswas et al. [22] consider the community organisation level. Finally, Camblong et al. [23] analyse the type of equipment implemented in order to respond to end-users' preferences. Unfortunately, in the reviewed publications the detail of the community is not considered and the solutions obtained do not specify the location of the equipment and the distribution combining microgrids and individual systems.

This paper aims to bridge this gap developing an optimisation model to design standalone electrification projects in rural areas of developing countries, including two sets of constraints to overcome the main limitations observed in yet implemented systems. In particular, five rural electrification projects in Peru and Bolivia are analysed, identifying that the management of the system should be eased and the security of supply should be improved. The inclusion of both issues can lead to projects better meeting end-users' preferences, but have not been studied at a community level in the reviewed design works. To include them in the design of stand-alone electrification systems, a new mathematical model is developed based on the procedure presented by Ferrer-Martí et 
al. [14]. Obtained solutions will then be appropriate to the technical, management and security of supply requirements of the community and its population, indicating the amount and location of all the equipment, the individual systems, the microgrids and their structure. Finally, the developed model is applied to the real community of Alto Peru (Peru). Results show that the model can help rural electrification promoters to carry out projects including some improvements that will lead to more sustainable and socially accepted systems that will widely compensate the obtained slight cost increases.

The rest of the paper is organised as follows. In Section 2 the stand-alone electrification systems are described and the performance of five real projects is analysed to justify the need of considering management and security of supply constraints in projects' design. In Section 3 the mathematical model is developed, including such constraints. In Section 4 the appropriateness of both constraints is validated through their use to design of a real community project. Finally, the main conclusions are summarised in Section 5.

\section{Definition of the problem}

In this section the stand-alone electrification systems generally used in rural areas of developing countries are described. For this purpose first the systems are technically defined and then the performance of real implemented projects is analysed identifying their main limitations and proposing some constraints to overcome them.

\subsection{System description}

The scheme of a hybrid wind-PV system with distribution through microgrids or individual systems is presented in Fig. 1. The electricity is produced by the wind turbines and/or the PV panels. The controllers protect batteries from overloads and deep discharges. The electricity is then stored in batteries to bridge the gap between generation and consumption. Next, the inverters transform the direct current from batteries into alternating current, which is more suitable for most electrical appliances. Finally, the electricity is distributed to consumption points (households, schools, health centres, etc.) as individual systems or radial microgrids [12]. Additionally, a meter is installed at each point to control its consumption.

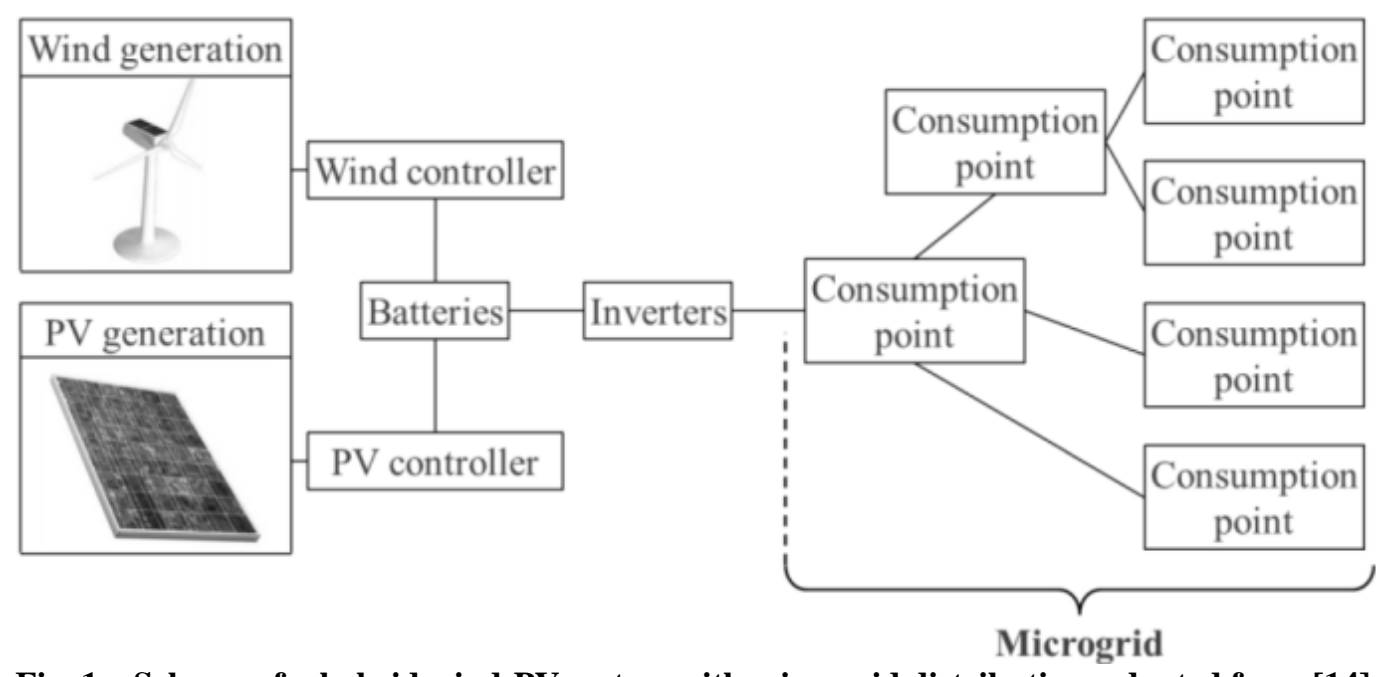

Fig. 1 - Scheme of a hybrid wind-PV system with microgrid distribution, adapted from [14] 
Complementarily, a management model is conceived according to the population characteristics to organise the community during the project lifetime $[9,24,25]$. Thus, educating population on an efficient electricity use and appropriate project management are key issues to ensure the project sustainability and end-users' reliability on electrification systems [26]. On the one hand, end-users are trained to realize domestic operation and maintenance tasks $[9,25]$. On the other hand, a technician is in charge of community operation and maintenance tasks and an administrator in charge of activities such as collecting end-users' monthly fees for later equipment repairs or substitutions $[24,25]$.

\subsection{Analysis of electrified communities}

Five electrified communities in rural areas of developing countries have been studied. Next their implemented stand-alone electrification systems are presented:

- Alto Peru, Peru [9]. Two wind microgrids, a PV microgrid and individual PV systems for the 26 users.

- Campo Alegre, Peru [9]. Hybrid wind-PV individual systems for the 20 users.

- El Alumbre, Peru [9]. Wind individual systems for the 35 users.

- Challapata, Bolivia [27]. Individual PV or wind systems for the 12 users.

- Turco, Bolivia [27]. Individual wind systems for the 13 users.

From the analysis of the performance of these electrification projects, the management of the system and the security of supply have been identified as key aspects to ensure the projects' sustainability.

\subsubsection{Analysis of the management of the electrification system}

The most influencing elements in the management of the electrification systems are:

- Conflicts due to the configuration of the electric distribution. As stated previously, microgrids offer several advantages in front of individual systems. Therefore its extension is recommended to be as much as possible. However, implementing too many microgrids or very small microgrids (in the same community), even more if combining with individual systems, can make the whole system too complex to manage. Thus, avoiding projects with too many microgrids and/or small microgrids is recommended.

- Differences in the paying rate. The fee that beneficiaries have to periodically pay for the electricity allows defraying the maintenance, repair and substitution of the equipment $[24,25]$. In the studied communities meters are installed in microgrid points to control their consumption and to adapt the fee to the real consumption. In contrast, in individual users meters are not installed to save costs and the paying rate is the same for any of them. However, this situation can lead to conflicts between users with different consumptions but paying the same rate or users with the same consumption but paying different rates. Thus, installing meters at all the points is suggested in order to establish a paying rate according to their real consumption.

Table 1 summarises the four proposed constraints to consider when designing electrification projects in order to ease the management of the system. 


\subsubsection{Analysis of the security of supply}

The security of supply is related to the type of equipment used in order to respond to users' needs (for instance, different technology, number or power of the generators). The most influencing elements are:

Table 1 - Constraints to ease the management of the system

\begin{tabular}{|l|l|}
\hline \multicolumn{1}{|c|}{ Limitation } & \multicolumn{1}{|c|}{ Constraint } \\
\hline \multirow{2}{*}{$\begin{array}{l}\text { Conflicts due to the } \\
\text { configuration of the } \\
\text { electric distribution. }\end{array}$} & $\begin{array}{l}\text { (MS1) Maximum number of individual users, to extend the } \\
\text { electrification through microgrids. }\end{array}$ \\
\cline { 2 - 3 } & $\begin{array}{l}\text { (MS2) Maximum number of microgrids, to avoid too many microgrids } \\
\text { in a same community. }\end{array}$ \\
\cline { 2 - 2 } & $\begin{array}{l}\text { MS3) Minimum number of users per microgrid, to reduce the effort } \\
\text { invested in management, not spending them in too small microgrids. }\end{array}$ \\
\hline $\begin{array}{l}\text { Differences in the } \\
\text { paying rate. }\end{array}$ & $\begin{array}{l}\text { (MS4) Meters only installed at microgrid points, to save costs, in front } \\
\text { of the natural option of installing them at all the consumption points. }\end{array}$ \\
\hline
\end{tabular}

- Variability of the energy resources. Although batteries supply electricity in periods without generation, if these periods extend longer than expected the energy supply may be cut off. Generally, the solar resource has a low variability over the time [28], while the wind resource is much more variable. Therefore each consumption point should be supplied by, at least, a certain amount of solar energy.

- Failures in the equipment. Most rural communities are very far from the cities, which greatly slows the repairs. Hence, promoting each consumption point to be supplied by more than one generator is recommended, so that if one fails, at least another one will still supply electricity.

- Differences in the energy allowance. As stated before, microgrids allow some flexibility in consumption (punctual increases in demand), while individual users cannot. This difference can lead to inequalities in the development opportunities. Therefore an additional amount of energy for individual users should be studied in order to compensate their disadvantages in front of microgrid users.

Table 2 summarises the three proposed constraints to consider when designing electrification projects in order to improve the security of supply.

Table 2 - Constraints to improve the security of supply

\begin{tabular}{|l|l|}
\hline \multicolumn{1}{|c|}{ Limitation } & \multicolumn{1}{c|}{ Constraint } \\
\hline $\begin{array}{l}\text { Variability of the } \\
\text { energy resources. }\end{array}$ & $\begin{array}{l}\text { (SS1) Minimum percentage of energy generated by PV panels at each generation } \\
\text { point, ensuring each point is supplied by a certain amount of solar resource. }\end{array}$ \\
\hline $\begin{array}{l}\text { Failures in the } \\
\text { equipment. }\end{array}$ & $\begin{array}{l}\text { (SS2) Minimum number of generation equipment at each generation point, so that } \\
\text { if one generator fails at least another one still supply the electricity. }\end{array}$ \\
\hline $\begin{array}{l}\text { Differences in the } \\
\text { energy allowance. }\end{array}$ & $\begin{array}{l}\text { (SS3) Additional percentage of energy on individual users, to compensate their } \\
\text { development disadvantages in front of microgrid users. }\end{array}$ \\
\hline
\end{tabular}

\section{Mathematical model}

The use of optimisation methods, as linear programming, is increasingly a powerful tool for solving real-life problems as the design of electrification systems using renewable 
energies [29]. This paper develops a new mixed integer linear programming model (Subsection 3.1), based on the procedure presented in [14]. The model is extended and adapted to include four constraints to ease the management of the system (Subsection 3.2) and three constraints to improve the security of supply (Subsection 3.3).

\subsection{Model with technical constraints}

Next the model with the technical constraints introduced in Subsection 2.1 is presented.

\subsubsection{Data}

- Consumption points

$P \quad$ Number of consumption points (households, schools, health centers). These are the points where the generators can be placed.

$L_{p d} \quad$ Distance $[\mathrm{m}]$ between two points $p$ and $d(p=1, \ldots, P ; d=1, \ldots, P)$.

$L_{\max } \quad$ Maximum length of a wire segment of the microgrid.

$Q_{p} \quad$ Set of points to which a point $p$ could be directly joined with a wire segment $\left(p=1, \ldots, P ; d=1, \ldots, P: p \neq d ; L_{p d} \leq L_{\max }\right)$.

$E D_{p} \quad$ Electric energy demand [Wh/day] at $p(p=1, \ldots, P)$.

$P D_{p} \quad$ Power demand [W] at $p$, considering the simultaneity factor $(p=1, \ldots, P)$.

- Electric generation

$A, N A$ Number of types of wind turbines $(a=1, \ldots, A)$ and maximum number of wind turbines that can be placed at a point, respectively.

$E A_{p a}$ Energy generated [Wh/day] by a wind turbine of type $a$ placed at $p(p=1, \ldots, P$; $a=1, \ldots, A)$.

$C A_{a} \quad$ Cost [US\$] of a wind turbine of type $a$, including the wind controller $(a=1, \ldots, A)$.

$S, N S$ Number of types of PV panels $(s=1, \ldots, S)$ and maximum number of PV panels that can be placed at a point, respectively.

$E S_{s} \quad$ Energy generated [Wh/day] by a PV panel of the type $s(s=1, \ldots, S)$.

$P S_{s} \quad$ Maximum power [W] of a PV panel of type $s(s=1, \ldots, S)$.

$C S_{s} \quad$ Cost [US\$] of a PV panel of type $s(s=1, \ldots, S)$.

$Z \quad$ Number of types of PV controllers $(z=1, \ldots, Z)$.

$P Z_{z} \quad$ Maximum power [W] of a PV controller of type $z(z=1, \ldots, Z)$.

$C Z_{z} \quad$ Cost [US\$] of a PV controller of type $z(z=1, \ldots, Z)$.

- Electric equipment

$B \quad$ Number of types of batteries $(b=1, \ldots, B)$.

$E B_{b} \quad$ Capacity [Wh] of a battery of type $b(b=1, \ldots, B)$.

$C B_{b} \quad$ Cost [US\$] of a battery of type $b(b=1, \ldots, B)$.

$\eta b \quad$ Efficiency of the batteries [fraction of unity].

$D B \quad$ Maximum proportion of discharge admitted in the batteries.

$D A \quad$ Required autonomy of the batteries [days].

I, NI Number of types of inverters $(i=1, \ldots, I)$ and maximum number of inverters that can be placed at a point, respectively.

$P I_{i} \quad$ Maximum power [W] of an inverter of type $i(i=1, \ldots, I)$.

$C I_{i} \quad$ Cost [US\$] of an inverter of type $i(i=1, \ldots, I)$.

$\eta i \quad$ Efficiency of the inverters [fraction of unity].

CM Cost [US\$] of an electric meter. 


\section{- Electric distribution}

$C \quad$ Number of types of wires $(c=1, \ldots, C)$.

$R C_{c} \quad$ Electric resistance (feed and return) $[\Omega / \mathrm{m}]$ of a wire of type $c(c=1, \ldots, C)$.

$I C_{c} \quad$ Maximum intensity $[\mathrm{A}]$ of a wire of type $c(c=1, \ldots, C)$.

$C C_{c}$ Cost [US $\left.\$ / \mathrm{m}\right]$ of a wire of type $c$, feed-return and infrastructure $(c=1, \ldots, C)$.

$V_{n} \quad$ Nominal voltage [V].

$V_{\text {min }} \quad$ Minimum voltage [V].

$V_{\max } \quad$ Maximum voltage [V].

$\eta c \quad$ Efficiency of the wires [fraction of unity].

\subsubsection{Variables}

- Integer non-negative variables

$x a_{p a} \quad$ Number of wind turbines of type $a$ placed at point $p(p=1, \ldots, P ; a=1, \ldots, A)$.

$x s_{p s} \quad$ Number of PV panels of type $s$ placed at point $p(p=1, \ldots, P ; s=1, \ldots, S)$.

$x z_{p z} \quad$ Number of PV controllers of type $z$ placed at point $p(p=1, \ldots, P ; z=1, \ldots, Z)$.

$x b_{p b} \quad$ Number of batteries of type $b$ placed at point $p(p=1, \ldots, P ; b=1, \ldots, B)$.

$x i_{p i} \quad$ Number of inverters of type $i$ placed at point $p(p=1, \ldots, P ; i=1, \ldots, I)$.

- Float non-negative variables

$f e_{p d} \quad$ Flow of energy [Wh/day] between the points $p$ and $d\left(p=1, \ldots, P ; d \in Q_{p}\right)$.

$f p_{p d} \quad$ Flow of power [W] between the points $p$ and $d\left(p=1, \ldots, P ; d \in Q_{p}\right)$.

$v_{p} \quad$ Voltage $[\mathrm{V}]$ at the point $\mathrm{p}\left(v_{p}=V_{\min }, \ldots, V_{\max } ; p=1, \ldots, P\right)$.

- Binary variables

$x g_{p} \in\{0,1\} \quad 1$, if and only if at least a wind turbine or PV panel is placed at point $p$ $(p=1, \ldots, P)$.

$x c_{p d c} \in\{0,1\} \quad 1$, if and only if there is a wire of type $c$ between the points $p$ and $d$ $\left(p=1, \ldots, P ; d \in Q_{p} ; c=1, \ldots, C\right)$.

\subsubsection{Objective function}

The objective function minimises the initial investment cost: wind turbines, PV panels, PV controllers, batteries, inverters, meters (at all the consumption points) and wires.

$$
\begin{aligned}
{[M I N] Z=} & \sum_{p=1}^{P} \sum_{a=1}^{A} C A_{a} \cdot x a_{p a}+\sum_{p=1}^{P} \sum_{s=1}^{S} C S_{s} \cdot x s_{p s}+\sum_{p=1}^{P} \sum_{z=1}^{Z} C Z_{z} \cdot x z_{p z}+\sum_{p=1}^{P} \sum_{b=1}^{B} C B_{b} \cdot x b_{p b}+ \\
& \sum_{p=1}^{P} \sum_{i=1}^{I} C I_{i} \cdot x i_{p i}+C M \cdot P+\sum_{p=1}^{P} \sum_{d \in Q_{p}} \sum_{c=1}^{C} L_{p d} \cdot C C_{c} \cdot x c_{p d c}
\end{aligned}
$$

\subsubsection{Constraints}

\section{- Electric generation and accumulation}

Constraints (2), (3) and (4) define the generation points $\left(x g_{p}=1\right)$ and limit the maximum number of wind turbines (2) and PV panels (3) that can be installed at a same point. Constraint (5) imposes the conditions of conservation and satisfaction of the energy demand: the energy arriving at a point $p$ plus the energy generated at the own $p$ must be 
higher (or equal) than the energy consumed by $p$ plus the energy leaving $p$. The constraint includes the efficiency of the batteries, the inverters and the wires. Constraint (6) is analogous to constraint (5), but for the power demand. The emplacement, type and quantity of inverters are determined according to users' demand and only considering the wires' efficiency. Constraint (7) forces batteries to store enough energy to cover the demand of the supplied users, considering the required days of autonomy and the discharge factor. In constraint (8), PV controllers must be adequately powered, according to the PV panels installed at the same point. Finally, constraint (9) forces inverters to be only placed at generation points.

$$
\begin{aligned}
& \sum_{a=1}^{A} x a_{p a} \leq N A \cdot x g_{p} \quad p=1, \ldots, P \\
& \sum_{s=1}^{S} x s_{p s} \leq N S \cdot x g_{p} \quad p=1, \ldots, P \\
& \sum_{a=1}^{A} x a_{p a}+\sum_{s=1}^{S} x s_{p s} \geq x g_{p} \quad p=1, \ldots, P \\
& \sum_{q=1 \mid p \in Q_{q}}^{P} f e_{q p}+\sum_{a=1}^{A} E A_{p a} \cdot x a_{p a}+\sum_{s=1}^{S} E S_{s} \cdot x s_{p s} \geq \\
& \frac{E D_{p}}{\eta b \cdot \eta i}\left(\frac{1}{\eta c}+\left(1-\frac{1}{\eta c}\right) x g_{p}\right)+\sum_{d \in Q_{p}} f e_{p d}
\end{aligned} \quad p=1, \ldots, P
$$

\section{- Electric distribution}

Constraints (10) and (11) respectively relate the energy and power flows with the existence of a wire. The condition of radial scheme of the microgrids is imposed in constraint (12): each point can have, at the most, one input wire except for the generation points that cannot have any. Constraint (13) establishes the voltage drop between two points, considering the type of wire used. Finally, constraint (14) defines that the intensity flowing between two points connected by a wire cannot be higher than the maximum admissible intensity of the type of wire.

$$
\begin{array}{ll}
f e_{p d} \leq\left(\sum_{j=1}^{P} \frac{E D_{j}}{\eta b \cdot \eta i \cdot \eta c}\right) \sum_{c=1}^{C} x c_{p d c} & p=1, \ldots, P ; d \in Q_{p} \\
f p_{p d} \leq\left(\sum_{j=1}^{P} \frac{P D_{j}}{\eta c}\right) \sum_{c=1}^{C} x c_{p d c} & p=1, \ldots, P ; d \in Q_{p}
\end{array}
$$




$$
\begin{array}{ll}
\sum_{q=1 \mid p \in Q_{q}}^{P} \sum_{c=1}^{C} x c_{q p c}+x g_{p} \leq 1 & p=1, \ldots, P \\
v_{p}-v_{d} \geq \frac{L_{p d} \cdot R C_{c} \cdot f p_{p d}}{V_{n}}-\left(V_{\max }-V_{\min }\right)\left(1-x c_{p d c}\right) & p=1, \ldots, P ; d \in Q_{p} ; c=1, \ldots, C \\
\frac{f p_{p d}}{V_{n}}-\left(\sum_{j=1}^{P} \frac{P D_{j}}{V_{\text {min }} \cdot \eta c}\right)\left(1-x c_{p d c}\right) \leq I C_{c} & p=1, \ldots, P ; d \in Q_{p} ; c=1, \ldots, C
\end{array}
$$

\subsection{Considerations to ease the management of the system}

Next the changes with respect to the model from Subsection 3.1 when including the four constraints to ease the management of the system are presented:

- (MS1) Maximum number of individual users.

- (MS2) Maximum number of microgrids.

- (MS3) Minimum number of users per microgrid.

- (MS4) Meters only installed at microgrid points.

\subsubsection{Data}

The next input parameters are included:

$U_{\text {ind }} \quad$ Maximum number of individual users.

$N_{\max } \quad$ Maximum number of microgrids.

$U_{\min } \quad$ Minimum number of users per microgrid.

\subsubsection{Variables}

The next binary variables are included:

$x m_{p} \in\{0,1\} \quad 1$, if and only if $p$ belongs to a microgrid $(p=1, \ldots, P)$.

$x m g_{p} \in\{0,1\} 1$, if and only if $p$ belongs to a microgrid and is a generation point $(p=1, \ldots, P)$.

$x f_{p d f} \in\{0,1\} \quad 1$, if and only if between the points $p$ and $d$ there is an energy flow directed towards the point $f\left(p=1, \ldots, P ; d \in Q_{p} ; f=1, \ldots, P\right)$.

\subsubsection{Objective function}

The sixth addend of the objective function (1) is modified only if considering the constraint MS4 (Meters only installed at microgrid points):

$$
\begin{aligned}
{[M I N] Z=} & \sum_{p=1}^{P} \sum_{a=1}^{A} C A_{a} \cdot x a_{p a}+\sum_{p=1}^{P} \sum_{s=1}^{S} C S_{s} \cdot x s_{p s}+\sum_{p=1}^{P} \sum_{z=1}^{Z} C Z_{z} \cdot x z_{p z}+\sum_{p=1}^{P} \sum_{b=1}^{B} C B_{b} \cdot x b_{p b}+ \\
& \sum_{p=1}^{P} \sum_{i=1}^{I} C I_{i} \cdot x i_{p i}+\sum_{p=1}^{P} C M \cdot x m_{p}+\sum_{p=1}^{P} \sum_{d \in Q_{p}} \sum_{c=1}^{C} L_{p d} \cdot C C_{c} \cdot x c_{p d c}
\end{aligned}
$$




\subsubsection{Constraints}

- MS1: Maximum number of individual users.

Constraints (15), (16) and (17) determine microgrid points, which have an input (15) or an output (16) wire, while individual systems have no input nor output wires (17). Constraint (18) forces a maximum bound of $U_{\text {ind }}$ of individual users.

$$
\begin{array}{ll}
\sum_{q=1 \mid p \in Q_{q}}^{P} \sum_{c=1}^{C} x c_{q p c} \leq x m_{p} & p=1, \ldots, P \\
\sum_{d \in Q_{p}} \sum_{c=1}^{C} x c_{p d c} \leq(P-1) \cdot x m_{p} & p=1, \ldots, P \\
\sum_{q=1 \mid p \in Q_{q}}^{P} \sum_{c=1}^{C} x c_{q p c}+\sum_{d \in Q_{p}} \sum_{c=1}^{C} x c_{p d c} \geq x m_{p} & p=1, \ldots, P \\
\sum_{p=1}^{P} x m_{p} \geq P-U_{\text {ind }} &
\end{array}
$$

- MS2: Maximum number of microgrids.

Constraints (15), (16) and (17) are included. Constraints (19) and (20) define microgrid generation points, which are generation points $\left(x g_{p}=1\right)$ with a meter $\left(x m_{p}=1\right)$. Each microgrid has one and only one generation point, so limiting microgrid generation points is the same as limiting the number of microgrids (21).

$$
\begin{array}{ll}
x g_{p}+x m_{p}-2 \geq 2 \cdot\left(x m g_{p}-1\right) & p=1, \ldots, P \\
x g_{p}+x m_{p}-2 \leq x m g_{p}-1 & p=1, \ldots, P \\
\sum_{p=1}^{P} x m g_{p} \leq N_{\max } &
\end{array}
$$

- MS3: Minimum number of users per microgrid.

Constraints (15), (16), (17), (19) and (20) are included. Constraint (22) establishes that the energy flow between two points is equal to the sum of the energy flows directed towards all the supplied points. Constraint (23) forces that, each point $p$ has an energy flow from another point $q$ with destiny to the own $p$, unless $p$ is a generation point. Constraints (24) and (25) add that the energy flows arriving to a point $p$ with destiny to another point $f$ will have to leave $p$. In constraint (26) the size of the microgrids is bounded. Each microgrid has its own generation point plus the supplied points (whose quantity is equal to the amount of energy flows leaving the generation point).

$$
\begin{array}{cl}
f e_{p d} \geq \sum_{f=1}^{P} x f_{p d f} \cdot \frac{E D_{f}}{\eta b \cdot \eta i \cdot \eta c} & p=1, \ldots, P ; d \in Q_{p} \\
\sum_{q=1 \mid p \in Q_{q}}^{P} x f_{q p p}+x g_{p}=1 & p=1, \ldots, P
\end{array}
$$




$$
\begin{array}{ll}
\sum_{q=1 \mid p \in Q_{q}}^{P} x f_{q p f}+x g_{p} \geq \sum_{d \in Q_{p}} x f_{p d f} & p=1, \ldots, P ; f=1, \ldots, P ; p \neq f \\
\sum_{q=1 \mid p \in Q_{q}}^{P} x f_{q p f} \leq \sum_{d \in Q_{p}} x f_{p d f} & p=1, \ldots, P ; f=1, \ldots, P ; p \neq f \\
\sum_{d \in Q_{p}} \sum_{f=1}^{P} x f_{p d f} \geq x m g_{p} \cdot\left(U_{\text {min }}-1\right) & p=1, \ldots, P
\end{array}
$$

- MS4: Meters only installed at microgrid points.

The objective function ( 1 ') is used, and constraints (15), (16) and (17) are added.

\subsection{Considerations to improve the security of supply}

Next the changes with respect to the model from Subsection 3.1 when including the three constraints to improve the security of supply are listed:

- (SS1) Minimum percentage of energy generated by PV panels.

- (SS2) Minimum number of generation equipment at each generation point.

- (SS3) Additional percentage of energy on individual users.

\subsubsection{Data}

The next input parameters are included:

SOL Minimum percentage [fraction of unity] of energy generated by PV panels at each generation point.

$E_{\min } \quad$ Minimum number of generation equipment at each generation point.

$E D_{\text {ind }}$ Additional energy percentage [fraction of unity] for supplying to the individual users with respect to the microgrid users.

\subsubsection{Variables}

The next float non-negative variable is included:

$e d_{p} \quad$ Energy [Wh/day] supplied to the point $p(p=1, \ldots, P)$.

\subsubsection{Objective function}

The objective function (1) does not change.

\subsubsection{Constraints}

- SS1: Minimum percentage of energy generated by PV panels.

Constraint (27) forces the energy demand of each consumption point to be partially covered by a certain percentage $S O L$ of PV panels. This constraint is an energy balance at the point $p$ only focusing on the solar energy. 


$$
\begin{aligned}
\sum_{q=\| p \in Q_{q}}^{P} f e_{q p} & +\sum_{s=1}^{S} E S_{s} \cdot x s_{p s} \geq \\
& S O L\left(\frac{E D_{p}}{\eta b \cdot \eta i}\left(\frac{1}{\eta c}+\left(1-\frac{1}{\eta c}\right) x g_{p}\right)+\sum_{d \in Q_{p}} f e_{p d}\right) \quad p=1, . ., P
\end{aligned}
$$

- SS2: Minimum number of generation equipment at each generation point.

Constraint (4) is replaced by (4'), which forces each consumption point to be supplied, at least, with a certain number $E_{\min }$ of generators (wind turbines or PV panels).

$$
\sum_{a=1}^{A} x a_{p a}+\sum_{s=1}^{S} x s_{p s} \geq E_{\min } \cdot x g_{p} \quad p=1, \ldots, P
$$

\section{- SS3: Additional percentage of energy on individual users.}

Variable $x m_{p}$ and constraints (15), (16) and (17) are included. Constraints (5'), (7') and (27') respectively replace constraints (5), (7) and (27). As observed, the part associated to the energy demand is replaced by the new variable $e d_{p}$. Constraint (28) establishes that if a point $p$ belongs to a microgrid $\left(x m_{p}=1\right)$ the variable $e d_{p}$ cannot be less than its demand, while if the point $p$ is individual $\left(x m_{p}=0\right)$ the variable $e d_{p}$ cannot be less than its demand plus an additional percentage.

$$
\begin{array}{lr}
\sum_{q=\| p \in Q_{q}}^{P} f e_{q p}+\sum_{a=1}^{A} E A_{p a} \cdot x a_{p a}+\sum_{s=1}^{S} E S_{s} \cdot x s_{p s} \geq e d_{p}+\sum_{d \in Q_{p}} f e_{p d} & p=1, \ldots, P \\
\sum_{b=1}^{B} E B_{b} \cdot x b_{p b}+\left(\frac{D A}{D B} \sum_{j=1}^{P} \frac{E D_{j}}{\eta b \cdot \eta i \cdot \eta c}\right)\left(1-x g_{p}\right) \geq \frac{D A}{D B}\left(\sum_{d \in Q_{p}} f e_{p d}+e d_{p}\right) & p=1, \ldots, P \\
\sum_{q=\| p \in Q_{q}}^{P} f e_{q p}+\sum_{s=1}^{S} E S_{s} \cdot x s_{p s} \geq S O L\left(e d_{p}+\sum_{d \in Q_{p}} f e_{p d}\right) & p=1, \ldots, P \\
e d_{p} \geq \frac{E D_{p}}{\eta b \cdot \eta i}\left(\frac{1}{\eta c}+\left(1-\frac{1}{\eta c}\right) x g_{p}+E D_{\text {ind }} \cdot x g_{p}\left(1-x m_{p}\right)\right) & p=1, \ldots, P
\end{array}
$$

Note that in constraint (7') the variable $e d_{p}$ replaces $\frac{E D_{p}}{\eta b \cdot \eta i}$, while in constraints (5') and (27') it replaces $\frac{E D_{p}}{\eta b \cdot \eta i}\left(\frac{1}{\eta c}+\left(1-\frac{1}{\eta c}\right) x g_{p}\right)$. The key to understand this difference can be found in constraint (28). If the variable $x g_{p}$ is 0 , the point $p$ is not a generation point and constraint ( $\left.7^{\prime}\right)$ has no effect (batteries are not installed at non-generation points). If the variable $x g_{p}$ is 1 , the variable $e d_{p}$ is replaced by $\frac{E D_{p}}{\eta b \cdot \eta i}$ plus a component associated to the additional percentage if the point $p$ is individual. This is the required value for the three constraints (5'), (7') and (27'). Finally, constraint (28) is non-linear, but the component $x g_{p} \cdot\left(1-x m_{p}\right)$ can be linearised replacing by an auxiliary variable $\left(\operatorname{aux}_{p}\right)$. Besides, constraints (29), (30) and (31) are added. 


$$
\begin{array}{ll}
x g_{p}+\left(1-x m_{p}\right) \leq 1+\operatorname{aux}_{p} & p=1, \ldots, P \\
x g_{p} \geq \operatorname{aux}_{p} & p=1, \ldots, P \\
\left(1-x m_{p}\right) \geq \operatorname{aux}_{p} & p=1, \ldots, P
\end{array}
$$

\section{Influence of the management and the security of supply constraints on the electrification solutions}

In this Section, the influence on projects' design of the previously proposed two sets of constraints is analysed. The validation is carried out in the real community of Alto Peru (Peru). Next, the community is described (Subsection 4.1), and then the obtained results are presented and discussed for the constraints aiming to ease the management of the system (Subsection 4.2) and aiming to improve the security of supply (Subsection 4.3).

\subsection{The community of Alto Peru}

Alto Peru is located in the Andean highlands, in the region of Cajamarca (Peru). The study focuses on a part of the community composed of 26 consumption points, which were electrified between 2009 and 2010 by the NGOs Practical Action (Peru), Engineering Without Borders (Catalonia and Valencia, Spain) and Green Empowerment (EEUU). Fig. 2 shows elevation and wind maps of the community, and the distribution of the 26 consumption points.
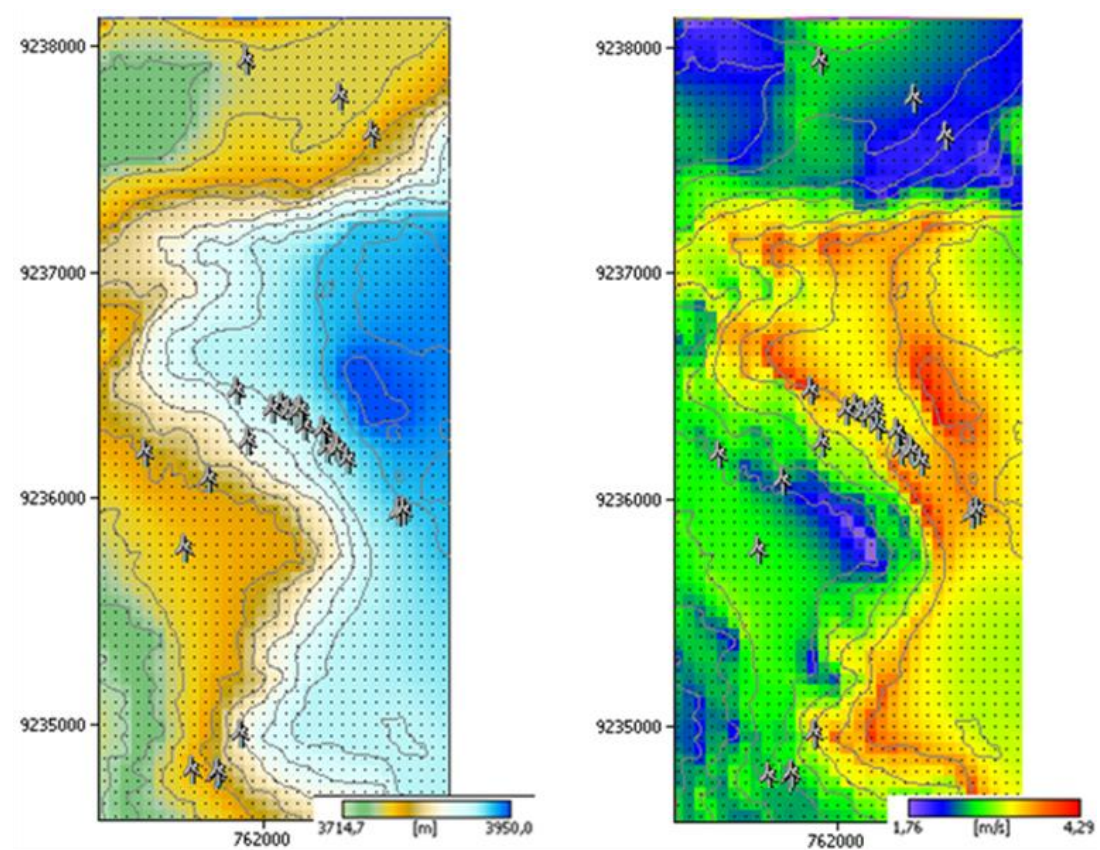

Fig. 2 - Elevation and wind maps of Alto Peru and location of the 26 consumption points

The equipment data used for designing the project is presented next:

- Demand: $280 \mathrm{Wh}$ /day of energy; $200 \mathrm{~W}$ of power; and 2 days of autonomy.

- Wind turbines (4 types). The energy is calculated from the wind map and varies from 42 to $6444 \mathrm{Wh} /$ day, depending on the point and the type of turbine considered. Cost: $\$ 1139$ to $\$ 5645$. Maximum number that can be installed at a same point: 3 . 
- Wind controllers. Included in the wind turbines.

- PV panels (4 types). Energy: 217 to $652 \mathrm{Wh} /$ day. Maximum power: 50 to $150 \mathrm{~W}$. Cost: $\$ 451$ to $\$ 1000$. Maximum number that can be installed at a same point: 30 .

- PV controllers (4 types). Maximum power: 50 to $200 \mathrm{~W}$. Cost: $\$ 67$ to $\$ 125$.

- Batteries (4 types). Capacity: 1500 to $3000 \mathrm{Wh}$. Cost: $\$ 225$ to $\$ 325$. Efficiency: 0.85 Discharge factor: 0.50

- Inverters (4 types). Maximum power: 300 to $3000 \mathrm{~W}$. Cost: $\$ 377$ to $\$ 2300$. Efficiency $85 \%$. Maximum number that can be installed at a same point: 30 .

- Meters (1 type). Cost: $\$ 50$.

- Wires (3 types). Resistance: 2.6 to $0.16 \Omega / \mathrm{km}$. Maximum intensity: 64 to $380 \mathrm{~A}$. Cost: $\$ 4.94$ to $\$ 5.79 / \mathrm{m}$. Efficiency: 0.91. Maximum length of a segment: $1000 \mathrm{~m}$.

- Nominal voltage: $220 \mathrm{~V}$. Minimum voltage: $210 \mathrm{~V}$. Maximum voltage: $230 \mathrm{~V}$.

A few months after the implementation of the project, the problems identified in Subsection 2.2 appeared. To overcome these limitations, the experts in charge of the project were interviewed, determining some values for each of the management and the security of supply constraints that would be appropriate if re-designing the project. These are presented in the following Subsections 4.2 and 4.3.

\subsection{Results and discussion: management of the system}

The values determined by the experts for the four constraints that aim to ease the management of the system in the community of Alto Peru are:

- (MS1) Maximum number of individual users. In the studied community there are 20 consumption points close to each other and 6 points further away (Fig. 2). A maximum of 6 individual users should be examined to tend to electrify the concentrated points with microgrids.

- (MS2) Maximum number of microgrids. In the implemented project 3 microgrids were installed and, especially for the two wind microgrids, some differences in the available energy appeared, generating disagreements between the users. Therefore, a maximum of one microgrid is proposed to be studied to avoid these differences.

- (MS3) Minimum number of users per microgrid. The more distant points stated previously could be gathered in two groups of 3 users each (Fig. 2). A minimum size of 3 users per microgrid should be analysed to ensure each group, if electrified with microgrids, is electrified together with a single microgrid.

- (MS4) Meters only installed at microgrid points. Comparing the installation of meters at all the consumption points and only at microgrid points is interesting for analysing the influence of these devices on the cost and the electrical configuration.

The presented model has to be solved with and without each value of the four constraints to analyse their influence on the solutions. Combining the inclusion or not of each value (to study the possible relations between them), $16\left(2^{4}=16\right)$ combinations have to be solved. However, two of them are repeated and not considered: when imposing a maximum of 1 microgrid (MS2) and a maximum of 6 individual users (MS1), the same solution is obtained whether or not imposing a minimum of 3 users per microgrid (MS3); both when installing meters at all the points or only at microgrid points (MS4). In some cases obtaining a solution from another just including or removing one of the values of the constraints is straightforward. For example once the 
solution installing meters at all the points has been obtained, a solution installing meters just at microgrid points can be easily calculated subtracting the meters cost of individual users (see solutions 1 vs. 2,3 vs. 4,5 vs. 6,7 vs. 8,9 vs. 10,11 vs. 12 and 13 vs. 14 , according to the nomenclature from Table 3). Moreover, solutions $9 / 10$ are valid for $5 / 6$ and $13 / 14$ as already accomplish the minimum of 3 users per microgrid (MS3). However it is worth solving the corresponding model because better solutions can be obtained.

Table 3 shows the results for the 14 combinations; solved with a maximum calculation time of 18000 seconds per combination (what is considered an acceptable time in literature [30]). The models are solved using specialised software (IBM ILOG CPLEX 12.2 Optimizer). For each solution the cost, the number of individual users, the number of microgrids, the number of users of each microgrid and the number of installed meters are shown. Moreover, Fig. 3 shows the obtained configurations for three representative solutions (3, 7 and 12). Triangles represent generation points (both for microgrids and individual systems) and circles represent points feed by a microgrid.

Table 3 - Results when easing the management of the system in Alto Peru

\begin{tabular}{|c|c|c|c|c|c|c|}
\hline & & & \multicolumn{2}{|c|}{ MS1: no limit } & \multicolumn{2}{|c|}{ MS1: 6 users } \\
\hline & & & MS4: all & MS4: mgrid & MS4: all & MS4: mgrid \\
\hline \multirow{12}{*}{$\begin{array}{c}\text { MS2: } \\
\text { no limit }\end{array}$} & \multirow{6}{*}{$\begin{array}{c}\text { MS3: } \\
\text { no limit }\end{array}$} & Solution $\mathbf{n}^{\circ}$ & 1 & 2 & 3 & 4 \\
\hline & & Cost $[\$]$ & 32509 & 32159 & 33222 & 32922 \\
\hline & & Indiv. users & 7 & 7 & 6 & 6 \\
\hline & & $\mathbf{N}^{0}$ mgrid & 3 & 3 & 3 & 3 \\
\hline & & Users / mgrid & $15 ; 2 ; 2$ & $15 ; 2 ; 2$ & $15 ; 3 ; 2$ & $15 ; 3 ; 2$ \\
\hline & & $\mathbf{N}^{\mathbf{0}}$ meters & 26 & 19 & 26 & 20 \\
\hline & \multirow{6}{*}{$\begin{array}{c}\text { MS3: } \\
3 \text { users/mgrid }\end{array}$} & Solution $\mathbf{n}^{\mathbf{0}}$ & 5 & 6 & 7 & 8 \\
\hline & & Cost $[\$]$ & 33001 & 32451 & 36147 & 35897 \\
\hline & & Indiv. users & 11 & 11 & 5 & 5 \\
\hline & & $\mathbf{N}^{\mathbf{0}}$ mgrid & 1 & 1 & 3 & 3 \\
\hline & & Users / mgrid & 15 & 15 & $15 ; 3 ; 3$ & $15 ; 3 ; 3$ \\
\hline & & $\mathbf{N}^{\mathbf{0}}$ meters & 26 & 15 & 26 & 21 \\
\hline \multirow{12}{*}{$\begin{array}{l}\text { MS2: } \\
1 \text { mgrid }\end{array}$} & \multirow{6}{*}{$\begin{array}{c}\text { MS3: } \\
\text { no limit }\end{array}$} & Solution $\mathrm{n}^{\mathbf{0}}$ & 9 & 10 & 11 & 12 \\
\hline & & Cost [\$] & 33001 & 32451 & 37526 & 37226 \\
\hline & & Indiv. users & 11 & 11 & 6 & 6 \\
\hline & & $\mathbf{N}^{\mathbf{0}}$ mgrid & 1 & 1 & 1 & 1 \\
\hline & & Users / mgrid & 15 & 15 & 20 & 20 \\
\hline & & $\mathbf{N}^{\mathbf{o}}$ meters & 26 & 15 & 26 & 20 \\
\hline & \multirow{6}{*}{$\begin{array}{c}\text { MS3: } \\
3 \text { users/mgrid }\end{array}$} & Solution $n^{0}$ & 13 & 14 & \multirow{6}{*}{ Idem 11} & \multirow{6}{*}{ Idem 12} \\
\hline & & Cost [\$] & 33001 & 32451 & & \\
\hline & & Indiv. users & 11 & 11 & & \\
\hline & & \begin{tabular}{|l|}
$N^{0}$ mgrid \\
\end{tabular} & 1 & 1 & & \\
\hline & & Users / mgrid & 15 & 15 & & \\
\hline & & $\mathrm{N}^{0}$ meters & 26 & 15 & & \\
\hline
\end{tabular}

Next, the influence on the electric distribution configuration and on the cost, of the four constraints to ease the management, is analysed; the solutions with and without each constraint are compared:

MS1. Some individual users are electrified through microgrids, by their adhesion to existing microgrids ( 1 vs. 3, 2 vs. 4, 9/13 vs. 11 and 10/14 vs. 12) or, moreover, by the creation of new microgrids (5 vs. 7 and 6 vs. 8). The cost increases between $2.2 \%$ and $14.7 \%$, the rise being higher if including the maximum of one microgrid (MS2). 


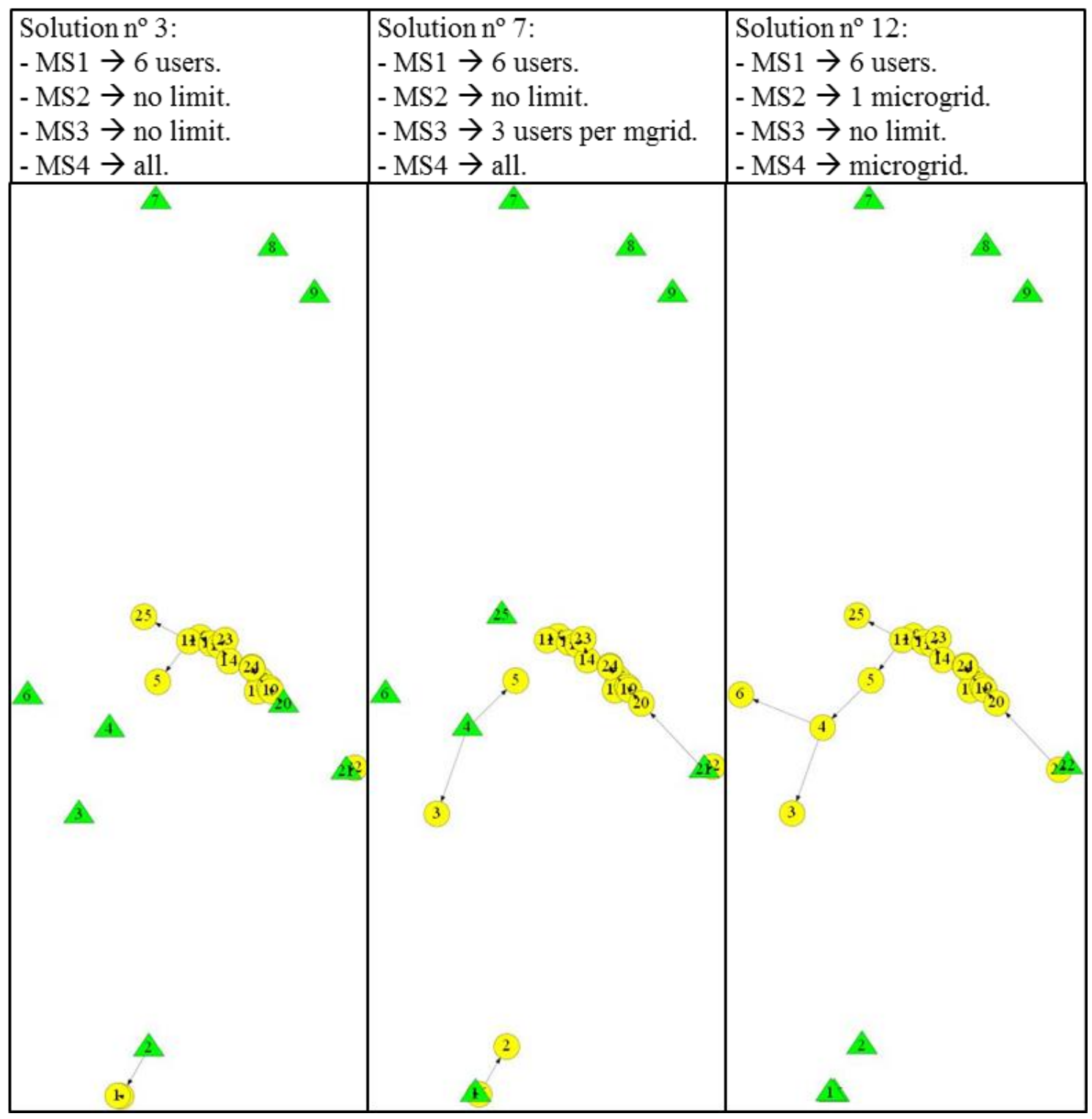

Fig. 3 - Configuration of the solutions 3, 7 and 12

MS2. The small microgrids are replaced by individual systems (1 vs. 9 and 2 vs. 10) or the big microgrid increases its size ( $3 / 7$ vs. 11 and 4/8 vs. 12). The cost increases between $0.9 \%$ and $1.5 \%$ if constraint MS1 is not included and between $13.0 \%$ and $13.1 \%$ if MS1 is included.

MS3. The two-user microgrids are replaced by individual systems ( 1 vs. 5 and 2 vs. 6 ) or by three-user microgrids ( 3 vs. 7 and 4 vs. 8), depending on the inclusion of constraint MS1. The cost increases between $0.9 \%$ and $9.0 \%$.

MS4. The influence of this constraint is very limited since the cost of meters is only $\$ 50$. The only observed effect is a reduction on the cost between $0.7 \%$ and $1.7 \%$.

Therefore, the difference in the cost between non-including and including each value of the studied constraints varies from $0.7 \%$ to $14.7 \%$. Moreover, when comparing the maximum cost solution to the minimum cost solution, there is a difference of $\$ 5367$ ( 2 vs. 11). Taking into account that there are 26 users, with a maximum investment of $\$ 206$ per user the management of the system can be eased. 


\subsection{Results and discussion: security of supply}

The values determined by the experts for the three constraints that aim to improve the security of supply in the community of Alto Peru are:

- (SS1) Minimum percentage of energy generated by PV panels. The region of the community of Alto Peru is a mountainous area, where the wind is very variable. A minimum of $25 \%$ of the energy generated by PV panels should be analysed to facilitate a more continuous supply (and avoid frequent electric cut offs).

- (SS2) Minimum number of generation equipment at each generation point. Alto Peru is far from the nearest city (Cajamarca) and even far from the capital of the country (Lima), where to repair the generation equipment or find some specific devices. Hence, this emplacement slows down any repair, and so a minimum of two pieces of generation equipment should be studied so that if one generator fails at least another one will still supply electricity.

- (SS3) Additional percentage of energy on individual users. Microgrids have several advantages in front of individual users. Therefore, a $40 \%$ of additional energy supplied to individuals should be examined to allow they have the same developing opportunities as, for example, use the energy for productive activities.

The proposed model has to be solved with and without each value of the four constraints to analyse their influence on the solutions. Combining the inclusion or not of each value (to study the possible relations between them), $8\left(2^{3}=8\right)$ combinations have to be solved. Table 4 shows the results for the 8 combinations. In this case, the cost is shown and the configuration is detailed specifying the generation equipment used for all the individual systems and the microgrids.

Table 4 - Results when improving the security of supply in Alto Peru

\begin{tabular}{|c|c|c|c|c|c|}
\hline & \multicolumn{2}{|c|}{ SS2: no limit } & \multicolumn{2}{|c|}{ SS2: 2} \\
\hline & & SS3: no limit & SS3: $40 \%$ & SS3: no limit & SS3: $40 \%$ \\
\hline \multirow{5}{*}{$\begin{array}{l}\text { SS1: } \\
\text { no } \\
\text { limit }\end{array}$} & Sol. $n^{\mathbf{o}}$ & 1 & 2 & 3 & 4 \\
\hline & Cost [\$] & 32509 & 34455 & 33594 & 35555 \\
\hline & \multirow{3}{*}{$\begin{array}{c}\text { Configuration } \\
\text { (generation } \\
\text { equipment) }\end{array}$} & $\begin{array}{l}7 \text { indiv users } \\
\text { (1 PV 100W) }\end{array}$ & $\begin{array}{l}7 \text { indiv users } \\
(1 \mathrm{PV} 150 \mathrm{~W})\end{array}$ & $\begin{array}{l}7 \text { indiv users } \\
(2 \mathrm{PV} 50 \mathrm{~W})\end{array}$ & $\begin{array}{l}5 \text { indiv users } \\
(1 \mathrm{PV} 50 \mathrm{~W} ; \\
1 \mathrm{PV} 75 \mathrm{~W})\end{array}$ \\
\hline & & $\begin{array}{c}2 \text { mgrid of } 2 \text { users } \\
\text { (1 PV 50W; } \\
1 \text { PV 150W) }\end{array}$ & $\begin{array}{c}2 \text { mgrid of } 2 \text { users } \\
\text { (1 PV 50W; } \\
1 \text { PV 150W) }\end{array}$ & $\begin{array}{c}2 \text { mgrid of } 2 \text { users } \\
\text { (1 PV 50W; } \\
1 \text { PV 150W) }\end{array}$ & $\begin{array}{c}3 \text { mgrid of } 2 \text { users } \\
\text { (1 PV 50W; } \\
1 \text { PV 150W) }\end{array}$ \\
\hline & & $\begin{array}{l}1 \text { mgrid of } 15 \text { users } \\
(1 \text { WT } 3500 \mathrm{~W})\end{array}$ & $\begin{array}{l}1 \text { mgrid of } 15 \text { users } \\
\text { (1 WT } 3500 W)\end{array}$ & $\begin{array}{c}1 \text { mgrid of } 15 \text { users } \\
\text { (1 PV 50W; } \\
1 \text { WT } 3500 W)\end{array}$ & $\begin{array}{c}1 \text { mgrid of } 15 \text { users } \\
\text { (1 PV 50W; } \\
1 \text { WT } 3500 W)\end{array}$ \\
\hline \multirow{5}{*}{$\begin{array}{l}\text { SS1: } \\
25 \%\end{array}$} & Solution $\mathbf{n}^{\mathbf{0}}$ & 5 & 6 & 7 & 8 \\
\hline & Cost [\$] & 35395 & 37462 & 36297 & 37909 \\
\hline & \multirow{3}{*}{$\begin{array}{c}\text { Configuration } \\
\text { (generation } \\
\text { equipment) }\end{array}$} & $\begin{array}{l}7 \text { indiv users } \\
(1 \mathrm{PV} 100 \mathrm{~W})\end{array}$ & $\begin{array}{l}6 \text { indiv users } \\
(1 \mathrm{PV} 150 \mathrm{~W})\end{array}$ & $\begin{array}{l}9 \text { indiv users } \\
(2 \mathrm{PV} 50 \mathrm{~W})\end{array}$ & $\begin{array}{l}4 \text { indiv users } \\
\text { (1 PV 50W; } \\
1 \mathrm{PV} 75 \mathrm{~W})\end{array}$ \\
\hline & & $\begin{array}{c}2 \text { mgrid of } 2 \text { users } \\
\text { (1 PV 50W; } \\
1 \text { PV 150W) }\end{array}$ & $\begin{array}{c}1 \text { mgrid of } 2 \text { users } \\
(1 \mathrm{PV} 50 \mathrm{~W} ; \\
1 \mathrm{PV} 150 \mathrm{~W})\end{array}$ & $\begin{array}{c}1 \text { mgrid of } 2 \text { users } \\
(1 \mathrm{PV} 50 \mathrm{~W} ; \\
1 \mathrm{PV} 150 \mathrm{~W})\end{array}$ & $\begin{array}{c}3 \text { mgrid of } 2 \text { users } \\
\text { (1 PV 50W; } \\
1 \text { PV 150W) }\end{array}$ \\
\hline & & $\begin{array}{c}1 \text { mgrid of } 15 \text { users } \\
\text { (1 PV } 75 \mathrm{~W} ; \\
2 \text { PV } 150 \mathrm{~W} ; \\
1 \text { WT } 3500 \mathrm{~W})\end{array}$ & $\begin{array}{l}1 \text { mgrid of } 18 \text { users } \\
\text { (3 PV } 150 W ; \\
1 \text { WT } 3500 W)\end{array}$ & $\begin{array}{c}1 \text { mgrid of } 15 \text { users } \\
\text { (1 PV } 75 W ; \\
2 \text { PV } 150 W ; \\
1 \text { WT } 3500 W)\end{array}$ & $\begin{array}{c}1 \text { mgrid of } 16 \text { users } \\
\text { (1 PV } 100 \mathrm{~W} ; \\
2 \text { PV } 150 \mathrm{~W} ; \\
1 \text { WT } 3500 \mathrm{~W})\end{array}$ \\
\hline
\end{tabular}


Next, the influence on the equipment typology and on the cost, of the three proposed constraints to improve the security of supply, is analysed; the solutions with and without each constraint are compared:

SS1. In the large microgrid a $3500 \mathrm{~W}$ wind turbine is always installed, but additional PV panels are added when including this constraint ( 1 vs. 5,2 vs. 6,3 vs. 7 and 4 vs. 8 ). The total cost increases between $6.6 \%$ and $8.9 \%$; and the extra energy is used for extending the microgrid including individual systems (4 vs. 8) and a two-user microgrid (2 vs. 6).

SS2. The PV panel used for individual users in solutions $1,2,5$ and 6 is substituted by 2 PV panels in solutions 3, 4, 7 and 8, because the total power is very similar. Moreover, a PV panel is added to the large microgrid when necessary (1 vs. 3 and 2 vs. 4 ). Given that PV panels have very low economies of scale, the cost increase is not very significant (between $1.2 \%$ and $3.3 \%$ ).

SS3. Individual points have a higher energy demand and so require more powerful equipment ( 1 vs. 2,3 vs. 4,5 vs. 6 and 7 vs. 8). Besides, in some cases some individual users are added to the large microgrid (5 vs. 6 and 7 vs. 8 ) and/or are grouped in twouser microgrids ( 3 vs. 4 and 7 vs. 8) since individual electrification is proportionally more expensive than microgrids. The cost increases between $4.4 \%$ and $6.0 \%$.

Therefore, the difference in the cost between non-including and including each value of the studied constraints varies from $1.2 \%$ to $8.9 \%$. Moreover, when comparing the maximum cost solution to the minimum cost solution, there is a difference of $\$ 5400$ (1 vs. 8). Taking into account that there are 26 users, with a maximum investment of $\$ 208$ per user the security of supply can be increased.

\section{Conclusions}

The aim of this work is to include management and security of supply constraints in the design of stand-alone hybrid wind-PV electrification systems for rural communities in developing countries. For this purpose the performance of five real electrification projects has been analysed. Thus two essential aspects of the solutions requiring to be improved have been identified: the management of the system and the security of supply. To overcome them, a mathematical model is developed including two sets of constraints to reflect the tendency in real projects. Therefore, the model obtains the minimum cost size and location of all the equipment, combining microgrids and individual systems, and considering the detail of the demand and the energy resources. To ease the management of the system the configuration of the electrical equipment is studied, analysing the amount, size and extension of the microgrids and the installation of meters. To improve the security of supply, their typology is examined through a minimum percentage of energy generated by PV panels, a minimum number of generation equipment and an additional percentage of energy on individual users. Finally, the two proposed sets of constraints are validated in the real community of Alto Peru (Peru), proving that the obtained solutions have many benefits that highly compensate the possible slight cost increases. 
For future research, it would be interesting to study the combination of the two sets of constraints. However, the models' solving process including both sets is expected to be complicated and very time consuming; especially if different electrification solutions are generated, in order to compare them and select the best one. Therefore we are currently working on a methodology that integrates the two sets of constraints, and uses a heuristic solving process to obtain good solutions in a shorter calculation time. Additionally, electrified communities in other countries or contexts could be analysed in order to identify new considerations that could be included in the projects' design. Special attention should be put on aspects leading to socially sustainable projects; i.e. mechanisms for a systems' appropriation and a community development ensuring responsible equipment maintenance and efficient and equitable use of electricity.

\section{Acknowledgements}

This paper was supported by the Centre of Cooperation for Development of the Universitat Politècnica de Catalunya (UPC). The authors are very grateful for all the assistance and support provided by the NGOs Practical Action (Peru), Engineering Without Borders (Catalonia, Spain), and Green Empowerment (USA). Additionally the authors would like to thank the anonymous reviewers for their valuable comments and suggestions to improve the quality of the paper.

\section{References}

[1] IEA, International Energy Association, 2011. World energy outlook.

[2] M. Kanagawa, T. Nakata, Assessment of access to electricity and the socio-economic impacts in rural areas of developing countries, Energy Policy 36 (2008) 2016-2029.

[3] A. Chaurey, M. Ranganathana, P. Mohanty, Electricity access for geographically disadvantaged rural communities - Technology and policy insights, Energy Policy 32 (2004) 1693-1705.

[4] M.F. Akorede, H. Hizam, E. Pouresmaeil, Distributed energy resources and benefits to the environment, Renew. Sustain. Energy Rev. 14 (2010) 724-734.

[5] M.K. Deshmukh, S.S. Deshmukh, Modeling of hybrid renewable energy systems, Renew. Sustain. Energy Rev. 12 (2008) 235-249.

[6] L. Ferrer-Martí, A. Garwood, J. Chiroque, R. Escobar, J. Coello, M. Castro, A community smallscale wind generation project in Peru, Wind Eng. 34 (2010) 277-288.

[7] J.A. Alzola, I. Vechiu, H. Camblong, M. Santos, M. Sall, G. Sow, Microgrids project Part 2: Design of an electrification kit with high content of renewable energy sources in Senegal, Renew. Energy 34 (2009) 2151-2159.

[8] C. Kirubi, A. Jacobson, D.M. Kammen, A. Mills, Community-based electric micro-grids can contribute to rural development: Evidence from Kenya, World Dev. 37 (2009) 1208-1221.

[9] L. Ferrer-Martí, A. Garwood, J. Chiroque, B. Ramírez, O. Marcelo, M. Garfí, E. Velo, Evaluating and comparing three community small-scale wind electrification projects. Renew. Sustain. Energy Rev. 16 (2012) 5379-5390.

[10] J.L. Bernal-Agustín, R. Dufo-Lopez, Simulation and optimization of stand-alone hybrid renewable energy systems. Renew. Sustain. Energy Rev. 13 (2009) 2111-2118.

[11] A.G. Ter-Gazarian, N. Kagan, Design model for electrical distribution systems considering renewable, conventional and energy storage units. IEE Proc. C 139 (1992) 499-504.

[12] T.W. Lambert, D.C. Hittle, Optimization of autonomous village electrification systems by simulated annealing, Sol. Energy 68 (2000) 121-132.

[13] A.K. Akella, M.P. Sharma, R.P. Saini, Optimum utilization of renewable energy sources in a remote area, Renew. Sustain. Energy Rev. 11 (2007) 894-908.

[14] L. Ferrer-Martí, B. Domenech, A. García-Villoria, R. Pastor, A MILP model to design hybridphotovoltaic isolated rural electrification projects in developing countries, Eur. J. Oper Res. 226 (2013) 293-300.

[15] M. Schäfer, N. Kebir, K. Neumann, Research needs for meeting the challenge of decentralized energy supply in developing countries, Energy Sustain. Dev. 15 (2011) 324-329. 
[16] J.J. Wang, Y.Y. Jing, C.F. Zhang, J.H. Zhao, Review on multi-criteria decision analysis aid in sustainable energy decision-making, Renew. Sustain. Energy Rev. 13 (2009) 2263-2278.

[17] M. Beccali, M. Cellura, M. Mistretta, Decision-making in energy planning. Application of the Electre method at regional level for the diffusion of renewable energy technology, Renew. Energy 28 (2003) 2063-2087.

[18] F. Cavallaro, L. Ciraolo, A multicriteria approach to evaluate wind energy plants on an Italian island, Energy Policy 33 (2005) 235-244.

[19] E. Georgopoulou, D. Lalas, L. Papagiannakis, A multicriteria decision aid approach for energy planning problems: The case of renewable energy option, Eur. J. Oper. Res. 103 (1997) 38-54.

[20] A.I. Chatzimouratidis, P.A. Pilavachi, Technological, economic and sustainability evaluation of power plants using the Analytic Hierarchy Process, Energy Policy 37 (2009) 778-787.

[21] J.A. Cherni, I. Dyner, F. Henao, P. Jaramillo, R. Smith, R. Olade, Energy supply for sustainable rural livelihoods. A multi-criteria decision-support system, Energy Policy 35 (2007) 1493-1504.

[22] W.K. Biswas, P. Bryce, M. Diesendorf, Model for empowering rural poor through renewable energy technologies in Bangladesh, Env. Sci. Policy 4 (2001) 333-344.

[23] H. Camblong, J. Sarr, A.T. Niang, O. Curea, J.A. Alzola, E.H. Sylla, M. Santos, Micro-grids project, Part 1: Analysis of rural electrification with high content of renewable energy sources in Senegal, Renew. Energy 34 (2009) 2141-2150.

[24] L. Ferrer-Martí, A. Garwood, J. Chiroque, R. Escobar, J. Coello, M. Castro. A community smallscale wind generation project in Peru, Wind Eng. 34 (2010) 277-288.

[25] R. Escobar, D. Vilar, E. Velo, L. Ferrer-Martí, B. Domenech, Promoting and improving renewable energy projects through local capacity development, in: A. Sencan-Sahin (Ed.), Modeling and optimization of renewable energy systems, E-Intech, 2012, pp. 147-170.

[26] B. Domenech, L. Ferrer-Martí, P. Lillo, R. Pastor, J. Chiroque. A community electrification project: Combination of microgrids and household systems fed by wind, PV or micro-hydro energies according to micro-scale resource evaluation and social constraints, Energy Sustain. Dev. 23 (2014) 275-285.

[27] L. Ferrer-Martí, B. Domenech, W. Canedo, C. Reza, M. Tellez, M. Dominguez, L. Perone, J. Salinas, Experiences of community wind electrification projects in Bolivia: evaluation and improvements for future projects, in: M. Nayeripour, M. Keshthi (Ed.), Sustainable growth and applications in renewable energy sources, E-Intech, 2011, pp. 85-106.

[28] C.A. Gueymard, S.M. Wilcox, Assessment of spatial and temporal variability in the US solar resource from radiometric measurements and predictions from models using ground-based or satellite data, Sol. Energy 85 (2011) 1068-1084.

[29] R. Baños, F. Manzano-Agugliaro, F.G. Montoya, C. Gil, A. Alcayde, J. Gómez, Optimization methods applied to renewable and sustainable energy: A review, Renew. Sustain. Energy Rev. 15 (2011) 1753-1766.

[30] L. Ferrer-Martí, R. Pastor, G.M. Capó, E. Velo, Optimizing microwind rural electrification projects. A case study in Peru, J. Glob. Optim. 50 (2011) 127-143. 